\title{
Increase in experimental infarct size with digoxin in a canine model of myocardial ischemia-reperfusion injury
}

\begin{abstract}
In the present study, dogs were pretreated with intravenous digoxin, $0.0125 \mathrm{mg} / \mathrm{kg} / \mathrm{day}$, for 6 to 7 consecutive days to achleve cllnically relevant serum concentrations; untreated animals were used as control subjects. After pretreatment, nine digoxin-pretreated dogs and nine control dogs were anesthetized and subjected to a 60 -minute occlusion of the left circumflex coronary artery, followed by 6 hours of reperfusion. Anatomic myocardial inferct size, expressed as a percentage of the areas at risk of infarction and as a percentage of the total left ventricle were:

$20.2 \pm 3.3 \%$ control vs $35.4 \pm 6.2 \%$ digoxin-pretreated $(p<0.05)$ and $8.6 \pm 1.3 \%$ control vs $14.7 \pm 2.5 \%$ digoxin-pretreated $(p<0.05)$, respectively $(2.04 \pm 0.37 \mathrm{ng} / \mathrm{mi}$ serum digoxin). Regional myocardial blood flow in the nonischemic and ischemic zones tended to be lower in digoxin-pretreated than in control animals at baseline testing and were eignificently reduced in the anterior subendocardial sites of digoxin-pretreated dogs during ischemia and reperfusion. These data suggest that an exacerbation or enhancement of myocardial. ischemial-reperfusion injury may occur in the presence of clinically observable serum digoxin concentrations. (Am HEART J 1988;115:1171.)
\end{abstract}

Joseph J. Lynch, Ph.D., Paul J. Simpson, Ph.D., Kim P. Gallagher, Ph.D., Thomas B. McClanahan, B.S., Karl A. Lee, and Benedict R. Lucchesi, Ph.D., M.D. Ann Arbor, Mich.

Previous investigation in this laboratory have examined the effects of clinically relevant serum digoxin concentrations, achieved by multiday pretreatment regimens, on the frequency and severity of ventricular arrhythmias occurring in canine models of myocardial ischemic injury. ${ }^{1-4}$ In conscious dogs with recent anterior myocardial infarction, digoxin pretreatment was associated with a significantly enhanced incidence of ventricular fibrillation and ischemic post infarction death in response to an acute ischemic episode occurring at a site remote from the area of previous myocardial injury. ${ }^{1}$ The digoxin-associated enhancement of ischemic postinfarction mortality was reduced significantly by the subacute ablation of the left stellate ganglion ${ }^{2}$ and by acute beta-adrenoceptor blockade with nadolol, ${ }^{3}$

From the Departments of Pharmacology, Physiology, and Surgery, and the Thoracic Surgery Research Laboratory, The University of Michigan Medical School.

Supported by National Institutes of Health, Heart, Lung, and Blood Institute grants HL-35325 and HL-05806.

Received for publication Dec. 3, 1987; accepted Dec. 12, 1988.

Reprint requests: Joseph J. Lynch, PhD, Assistant Research Scientist, M6322 Medical Science Building I, Department of Pharmacology, The University of Michigan Medical School, Ann Arbor, MI 48109. suggesting an involvement of sympathetic nervous activity in the arrhythmogenic actions of "therapeutic" digoxin in this preparation. In a recent study, using conscious dogs without previous myocardial ischemic injury, the in situ development of acute posterolateral ischemia in the presence of clinically relevant serum digoxin concentrations resulted in the development of ventricular ectopic activity at 1 to 3 days after infarction that was less sensitive to suppression with conventional antiarrhythmic therapy compared to control animals subjected to posterolateral myocardial ischemia and infarction but not pretreated with digoxin. ${ }^{4}$ In the latter study, an examination of posterolateral myocardial infarct sizes at the conclusion of antiarrhythmic drug testing suggested that larger myocardial infarctions, expressed as a percentage of the total left ventricle, had evolved in the digoxin-pretreated group. ${ }^{4}$

In the present study, the effects of clinically relevant serum concentrations of digoxin, achieved by a 6- to 7-day pretreatment regimen, on the extent of developing myocardial infarction were evaluated in an anesthetized canine model of regional myocardial ischemia-reperfusion injury. In this preparation, the duration of regional ischemic injury was 
controlled and equivalent in both the digoxinpretreated and control groups, and the resulting myocardial infarct sizes were quantitated in terms of the total left ventricle and the area at risk of myocardial infarction. Regional myocardial blood flow was determined with the use of radiotracerlabeled microspheres in nonischemic and ischemic areas of myocardium to determine whether equivalent severities of ischemia were present in the digoxin-pretreated and control groups and to ascertain the effects of digoxin pretreatment on regional myocardial blood flow.

\section{METHODS}

Experimental groups. Nineteen male mongrel dogs were randomly assigned to either a treatment group that reccived intravenous digoxin $(0.0125 \mathrm{mg} / \mathrm{kg} / \mathrm{day}, \mathrm{n}=10$, $14.1 \pm 0.6 \mathrm{~kg})$ or a control group that received no intervention $(\mathbf{n}=9,15.7 \pm 1.0 \mathrm{~kg})$. In digoxin-treated animals, the drug was given as a peripheral intravenous infusion into a radial foreleg vein on six to seven consecutive afternoons during the pretreatment period. After the pretreatment period and immediately before the induction of anesthesia (see below), peripheral blood samples were obtained for determination of serum electrolyte concentrations in digoxin-pretreated and control animals and for determination of serum digoxin concentrations in digoxin-pretreated animals.

Surgical preparation. Digoxin-pretreated and control animals were anesthetized with Dial-urethane solution $(40 \%$ ethylurea, $10 \%$ allobarbital, $40 \%$ urethane; 0.6 $\mathrm{ml} / \mathrm{kg}$ intravenously), and the lungs were ventilated with room air by means of a cuffed endotracheal tube and a Harvard respirator. Cannulae were inserted into the left common carotid and right femoral arteries for withdrawal of reference arterial samples for determinations of myocardial blood flow; the left femoral artery was cannulated for measurement of systemic arterial pressure. A leftsided thoracotomy was performed in the fifth intercostal space, and the heart was suspended in a pericardial cradle. Catheters were placed into the left atrium for measurement of left atrial pressure and for injection of radiolabeled microspheres for measurement of regional blood flow. The proximal left circumflex coronary artery was dissected free to allow placement of an electromagnetic flow probe (Carolina Medical Electronics, King, N. C.) for measurement of coronary artery blood flow. Lead II ECG, systemic arterial pressure, left atrial pressure, and left circumflex coronary artery blood flow were recorded on a model 7 polygraph (Grass Instruments, Quincy, Mass.).

After a stabilization period of 30 minutes and injection of radiolabeled microspheres for determination of baseline regional myocardial blood flow, a critical stenosis of silk suture was applied to the proximal left circumflex coronary artery such that the maximum hyperemic response to a 10-second occlusion of the coronary artery was attenuated approximately $50 \%$, whereas basal coronary artery blood flow was unaffected. Regional posterolateral myocardial ischemia was then produced by occluding the proximal left circumflex coronary artery for 60 minutes by means of a silicone tubing snare, followed by restoration of blood flow for a period of 6 hours. The critical stenosis was removed after 30 minutes of reperfusion. The critical stenosis was used in the present study to reduce the incidence of ventricular fibrillation during the early reperfusion period. ${ }^{5}$

Postmortem determination of infarct size. After the 6-hour reperfusion period, the anesthetized dogs were killed by applying a supramaximal electric current to the surface of the left ventricle for induction of ventricular fibrillation. Each heart was excised rapidly and prepared for quantification of infarct size. A dual-perfusion technique was used to determine the size of the infarcted mass, which was expressed as a percentage of the anatomic area at risk of infarction and as a percentage of the total left ventricle ${ }^{6 \cdot 9}$ Cannulae were placed in the aorta above the coronary ostia and in the left circumflex coronary artery at the site of the occlusion. The left circumflex coronary artery bed was perfused with $1.5 \%$ triphenyltetrazolium chloride in $0.1 \mathrm{~mol} / \mathrm{L}$ phosphate buffer $\left(\mathrm{pH} 7.4,37^{\circ} \mathrm{C}\right)$ at a pressure of $100 \mathrm{~mm} \mathrm{Hg}$. Simultaneously, the aorta was perfused with $0.5 \%$ Evans blue dye at the same perfusion pressure to delineate the nonischemic myocardium. After 5 minutes of dual perfusion, the heart was cut into five sections perpendicular to the apex-base axis. The myocardium perfused by the left circumflex coronary artery constituted the area at risk of infarction and was identified by the presence of a red coloration (because of the reduction of triphenyltetrazolium to a red formazan by dehydrogenase enzymes in viable cells) and the absence of blue dye. The infarcted area was apparent as tissue that lacked staining (because of the loss of dehydrogenase activity in irreversibly injured tissue) within the red area at risk. ${ }^{9}$ The sections of left ventricle were traced onto a clear plastic overlay, and planimetry was performed to quantitate the size of the anatomic area at risk (as a percentage of the left ventricle) and the size of infarction (as percentages of the area at risk and the left ventricle) by means of an Apple IIE microcomputer and digitizing board. It is recognized that the simultaneous perfusion of both normal and ischemic myocardium at a pressure that exceeds the perfusion pressure of the ischemic zone in vivo may overestimate the area at risk of infarction. Also, some controversy exists regarding the correlation between the detection of irreversibly damaged tissue by means of triphenyltetrazolium staining as opposed to functional measurements. ${ }^{10}$ However, in the present study, there was good agreement between the delineation of the ischemic zone by measurements of blood flow and delineation by the dual-perfusion technique, suggesting that the degree of error was small. Previous studies also have shown an excellent correlation between triphenyltetrazolium staining for quantification of infarct size and results of histologic examination of infarcts. ${ }^{6,9.11,12}$ Finally, any error that is encountered with the use of this technique for determi- 
nation of infarct size should be similar in both treatment groups, because the same technique was used in each experiment.

Regional myocardial blood flow measurements. Regional myocardial blood flow was measured with $15 \mu \mathrm{m}$ diameter radiotracer-labeled microspheres (New England Nuclear, North Billerica, Mass., and 3M Corp., St. Paul, Minn.) by the reference withdrawal method. ${ }^{13}$ Regional myocardial blood flow was determined at four time points during the experiment (baseline, 50-minute occlusion, and 1-hour and 4-hour reperfusion) by the randomly ordered injection of one of the following isotope-labeled microspheres at each time point: ${ }^{141} \mathrm{Ce},{ }^{46} \mathrm{Sc}$, ${ }^{113} \mathrm{Sn}$, or ${ }^{85} \mathrm{Sr}$. Approximately one to two million microspheres were injected into the left atrium at each time point. Reference carotid and femoral arterial samples were obtained at a constant rate of $7.05 \mathrm{ml} / \mathrm{min}$ with a Harvard withdrawal pump (Harvard Apparatus, Millis, Mass.) The withdrawal of reference blood samples was initiated before the injection of microspheres and was completed 90 seconds after flushing with $10 \mathrm{ml}$ saline solution after injection of the microspheres. Each vial of microspheres was mixed by sonication and vortex agitation before injection, and aliquots of the microsphere suspension were examined periodically under a microscope to ensure that adequate dispersal was being achieved.

Three basal transverse rings of left ventricle were used for determinations of regional myocardial blood flow after overlay tracings were made for determinations of infarct size. Full-thickness sections were obtained from the left circumflex central ischemic and left anterior control nonischemic areas. Each section of tissue was divided into three pieces (subendocardial, midmyocardial, and subepicardial) of equal thickness and mass (approximately 1 $\mathrm{gm}$ ). Tissue samples were placed in counting vials for the counting of radioactivity in a gamma scintillation counter (Tracor model 1185, Elk Grove, Ill.). After the counts in each tissue sample were corrected for background and overlapping counts, blood flow was calculated with the equation $\mathrm{Qm}=(\mathrm{Cm} \times \mathrm{Qr}) / \mathrm{Cr}$, where $\mathrm{Qm}=$ myocardial blood flow (ml/min), $\mathrm{Cm}=$ counts $/ \mathrm{min}$ in tissue samples, $\mathrm{Qr}=$ withdrawal rate of the reference arterial samples ( $\mathrm{ml} / \mathrm{min}$ ), and $\mathrm{Cr}=$ counts $/ \mathrm{min}$ in the reference arterial sample. Flow per gram of tissue $(\mathrm{ml} / \mathrm{min} / \mathrm{gm}$ ) was calculated by dividing the tissue sample flow by the sample mass. Background and overlap corrections and blood flow calculations were performed on an Apple II Plus microcomputer.

Exclusion criteria. The measurement of regional myocardial blood flow with radiotracer-labeled microspheres permits an accurate assessment of ischemic collateral blood flow during coronary artery occlusion. Previous studies $^{7,14}$ have shown a critical dependence of the extent of evolving myocardial infarction on the magnitude of regional myocardial blood flow in ischemic myocardium, and hence the necessity to ascertain and control for abnormally high ischemic regional myocardial blood flow resulting from excessive collateralization in experimental myocardial infarct size determinations. In the present study, a total of 19 dogs (10 digoxin pretreated and nine control) were entered into the experimental protocol. During postmortem assessment of regional myocardial blood flow in the central ischemic region, one digoxinpretreated dog was determined to have maintained an excessively high degree of regional myocardial blood during coronary artery occlusion compared to both the remaining cohort of digoxin-pretreated dogs $(n=9)$ and the untreated control group $(n=9)$. During left circumflex coronary artery occlusion, subendocardial blood flow in the central ischemic region of the aforementioned "high-flow" animal was $0.192 \mathrm{ml} / \mathrm{min} / \mathrm{gm}$ compared to subendocardial flow values of $0.04 \pm 0.01$ and $0.03 \pm 0.01$ $\mathrm{ml} / \mathrm{min} / \mathrm{gm}$ in the remaining digoxin-pretreated and control animals, respectively. Transmural myocardial blood flow in the "high-flow" animal during coronary artery occlusion was $0.322 \mathrm{ml} / \mathrm{min} / \mathrm{gm}$ compared to transmural blood flow values of $0.07 \pm 0.02$ and $0.06 \pm 0.01 \mathrm{ml} /$ $\mathrm{min} / \mathrm{gm}$ in the remaining digoxin-pretreated and control animals, respectively. The large magnitude of difference in regional blood flow values for the one digoxinpretreated "high-flow" animal and the remaining cohort of nine digoxin-pretreated animals strongly suggests that the high values for regional myocardial blood flow during coronary artery occlusion observed in the one animal were not the result of a specific treatment effect but rather the result of excessive collateralization in that one animal. Therefore, the data for the one digoxin-pretreated animal with high values for ischemic collateral myocardial blood flow have been excluded from consideration and, unless otherwise indicated, analyses of the data on hemodynamic and myocardial infarct size presented in this report are based on the results obtained from nine digoxinpretreated and nine untreated control animals.

Determination of serum digoxin and serum electrolyte concentrations. Blood samples for determination of serum digoxin, sodium, and potassium concentrations were ohtained at the end of the pretreatment period and immediately before the induction of anesthesia (approximately 16 hours after the preceding drug administration in the digoxin-pretreated group). For animals in the digoxin-pretreated group, venous blood samples were obtained from a radial vein not used previously for drug administration. Serum sodium and potassium concentrations were determined for the digoxin-pretreated and control animals by means of a flame photometer (model IL-143, Instrumentation Laboratory Inc, Boston, Mass.). Serum digoxin concentrations were determined in the digoxin-pretreated group by means of a radioimmunoassay technique in the Ligand Assay Laboratory at The University of Michigan Medical School, with the use of sheep antidigoxin antiserum.

Analysis of data. Hemodynamic data (heart rate, systemic arterial pressure, left atrial pressure, and left circumflex coronary artery blood flow) were analyzed at seven time points: (1) baseline, (2) 15 minutes after left circumflex coronary artery occlusion, (3) 50 minutes after 
Table I. Hemodynamic data at baseline, during left circumflex coronary artery occlusion, and after left circumflex coronary artery reperfusion

\begin{tabular}{|c|c|c|c|c|c|c|c|}
\hline \multirow{2}{*}{$\begin{array}{c}\text { Hemo- } \\
\text { dynamic } \\
\text { data }\end{array}$} & \multirow{2}{*}{$\begin{array}{c}\text { Base- } \\
\text { line }\end{array}$} & \multicolumn{2}{|c|}{ LCx occlusion } & \multicolumn{4}{|c|}{$L C x$ reperfusion } \\
\hline & & $15 \min$ & $50 \mathrm{~min}$ & $15 \mathrm{~min}$ & $1 \mathrm{hr}$ & $4 \mathrm{hr}$ & $6 \mathrm{hr}$ \\
\hline \multicolumn{8}{|c|}{ Heart rate (beats/min) } \\
\hline CNT & $139 \pm 6$ & $143 \pm 7$ & $144 \pm 7$ & $129 \pm 5$ & $132 \pm 6$ & $147 \pm 8$ & $153 \pm 11$ \\
\hline DIG & $152 \pm 10$ & $147 \pm 9$ & $147 \pm 8$ & $136 \pm 8$ & $137 \pm 8$ & $146 \pm 11$ & $149 \pm 12$ \\
\hline \multicolumn{8}{|c|}{ Mean arterial pressure (mm Hg) } \\
\hline CNT & $106 \pm 6$ & $95 \pm 6^{*}$ & $95 \pm 7^{*}$ & $88 \pm 5^{* *}$ & $87 \pm 5^{* *}$ & $87 \pm 6^{* *}$ & $87 \pm 7^{* *}$ \\
\hline DIG & $98 \pm 6$ & $86 \pm 6^{* *}$ & $81 \pm 6^{* *}$ & $73 \pm 4^{* *} \dagger$ & $78 \pm 4^{* *}$ & $81 \pm 4^{* *}$ & $72 \pm 4^{* *}$ \\
\hline \multicolumn{8}{|c|}{ Rate-pressure product $\left(\mathrm{HR} \times \mathrm{MAP}, \times 10^{3}\right)$} \\
\hline CNT & $15.0 \pm 1.3$ & $13.7 \pm 1.2$ & $13.9 \pm 1.4$ & $11.5 \pm 0.9^{* *}$ & $11.5 \pm 0.9^{* *}$ & $12.8 \pm 1.2$ & $13.7 \pm 2.0$ \\
\hline DIG & $15.2 \pm 1.7$ & $12.9 \pm 1.4$ & $12.3 \pm 1.5^{*}$ & $10.0 \pm 1.0^{* *}$ & $10.7 \pm 0.9^{* *}$ & $12.0 \pm 1.4^{* *}$ & $11.1 \pm 1.3^{* *}$ \\
\hline \multicolumn{8}{|c|}{ Left atrial pressure $(\mathrm{mm} \mathrm{Hg})$} \\
\hline CNT & $7.7 \pm 1.2$ & $8.7 \pm 0.9$ & $10.3 \pm 1.2$ & $7.4 \pm 0.8$ & $6.6 \pm 1.0$ & $7.4 \pm 0.8$ & $6.6 \pm 1.0$ \\
\hline DIG & $7.3 \pm 1.1$ & $8.8 \pm 1.5$ & $8.3 \pm 1.6$ & $7.3 \pm 0.7$ & $8.4 \pm 1.5$ & $7.3 \pm 0.7$ & $8.4 \pm 1.5$ \\
\hline \multicolumn{8}{|c|}{ LCx blood flow $(\mathrm{ml} / \mathrm{min})$} \\
\hline CNT & $16.6 \pm 1.6$ & $0^{* *}$ & $0^{* *}$ & $15.1 \pm 1.1$ & $13.7 \pm 1.1$ & $12.7 \pm 2.0$ & $9.9 \pm 0.9^{* *}$ \\
\hline DIG & $13.7 \pm 1.5$ & $0^{* *}$ & $0^{* * *}$ & $14.7 \pm 2.1$ & $12.8 \pm 1.5$ & $8.4 \pm 1.1^{* *}$ & $7.6 \pm 1.1^{* *}$ \\
\hline
\end{tabular}

Data are mean $\pm \mathrm{SE}$; for all parameters, $n=9$.

$\mathrm{CNT}=$ Untreated control group; $\mathrm{DIG}=$ digoxin-pretreated group; $\mathrm{HR}=$ heart rate; $\mathrm{LCx}=$ left circumflex coronary artery; $\mathrm{MAP}=$ mean arterial pressure.

${ }^{*} p<0.05$ and ${ }^{* *} p<0.01$ compared to appropriate baseline value within group (repeated-measures analysis of variance followed by Dunnett's test for multiple comparisons). $\nmid p<0.05$ comparing DIG vs CNT parameters at specified time point (unpaired Student's $t$ test).

coronary artery occlusion, (4) 15 minutes after coronary artery reperfusion, (5) 1 hour after coronary artery reperfusion, (6) 4 hours after coronary artery reperfusion, and (7) 6 hours after coronary artery reperfusion. A ratepressure product (heart rate $\times$ mean arterial pressure) was calculated at each time point as an index of myocardial oxygen consumption., ${ }^{75}$ Regional myocardial blood flow was determined at: (1) baseline, (2) 50 minutes after left circumflex coronary artery occlusion, (3) 1 hour after coronary artery reperfusion, and (4) 4 hours after coronary artery reperfusion. For the hemodynamic and regional blood flow data, comparisons for a given parameter within a group relative to the baseline value were made by means of a repeated-measures analysis of variance, followed by a Dunnett's test for multiple comparisons to one control (baseline) value. Comparisons between the two treatment groups at a given time point were made by means of a two-tailed, unpaired Student's $t$ test. Comparisons between the two groups for infarct size also were made by means of the two-tailed unpaired Student's $t$ test. The incidences of ventricular fibrillation in the digoxin pretreatment and control groups were compared by means of Fisher's exact test for binomial distribution. For evaluation of direct relationships between myocardial infarct size and hemodynamic or blood flow parameters, linear regression was performed and correlation coefficients were evaluated. The significance of the regression coefficient was tested by the calculation of a probability $(p)$ value, and $95 \%$ confidence intervals were determined for mean $(x, y)$ values for the regression lines to determine overlap. For all comparisons, $p<0.05$ was considered statistically significant. All data are expressed as mean \pm standard error.

\section{RESULTS}

Serum digoxin and electrolyte concentrations. Serum digoxin concentrations determined in the digoxin-pretreated animals were $2.04 \pm 0.37 \mathrm{ng} / \mathrm{ml}$ after a mean treatment duration of $6.7 \pm 0.3$ days. Serum sodium (125.4 \pm 1.5 and $119.9 \pm 3.0 \mathrm{mEq} / \mathrm{L})$ and potassium $(4.35 \pm 0.10$ and $4.25 \pm 0.12 \mathrm{mEq} / \mathrm{L})$ concentrations were similar in the control and digoxin-pretreated groups, respectively.

Hemodynamic parameters. Hemodynamic data are summarized in Table I. At baseline and during the period of left circumflex coronary artery occlusion, no significant between-group differences in measured hemodynamic parameters or in calculated rate-pressure product were noted for the digoxinpretreated and control groups, although the baseline value for left circumflex coronary artery blood flow tended to be reduced in the digoxin-pretreated group ( $-18 \%$ of control values; $p=0.20$ ) compared to the control group value (Table I). Both treatment groups displayed similar hemodynamic profiles in response to left circumflex coronary artery reperfusion after regional myocardial ischemia, with the only significant between-group difference being a reduction in mean arterial pressure in the digoxinpretreated group relative to the control value $(p<0.05)$ at the 15 -minute postreperfusion time point (Table I). During the reperfusion period, within-group comparisons to baseline values indicated progressive and significant declines in mean 
Table II. Myocardial blood flow in the central ischemic area (left circumflex distribution) at baseline, during left circumflex coronary artery occlusion, and after left circumflex coronary artery reperfusion

\begin{tabular}{|c|c|c|c|c|}
\hline & \multirow[b]{2}{*}{ Baseline } & \multirow{2}{*}{$\frac{\begin{array}{c}L C x \\
\text { occlusion }\end{array}}{50 \min }$} & \multicolumn{2}{|c|}{$\begin{array}{c}L C x \\
\text { reperfusion }\end{array}$} \\
\hline & & & $1 \mathrm{hr}$ & A hr \\
\hline \multicolumn{5}{|c|}{ Subendocardial MBF (ml/min/gm) } \\
\hline CNT & $0.62 \pm 0.05$ & $0.03 \pm 0.01^{* *}$ & $0.77 \pm 0.14$ & $0.38 \pm 0.05^{*}$ \\
\hline DIG & $0.56 \pm 0.07$ & $0.04 \pm 0.01^{* *}$ & $0.60 \pm 0.10$ & $0.29+0.04^{* *}$ \\
\hline \multicolumn{5}{|c|}{ Midmyocardial MBF (ml/min/gm) } \\
\hline CNT & $0.61 \pm 0.04$ & $0.05 \pm 0.02^{* *}$ & $0.58 \pm 0.09$ & $0.42 \pm 0.07$ \\
\hline DIG & $0.57 \pm 0.07$ & $0.06 \pm 0.02^{* *}$ & $0.59 \pm 0.06$ & $0.30+0.03^{* *}$ \\
\hline \multicolumn{5}{|c|}{ Subepicardial MBF (ml/min/gm) } \\
\hline CNT & $0.54 \pm 0.03$ & $0.08 \pm 0.02^{* *}$ & $0.42 \pm 0.04$ & $0.40 \pm 0.05$ \\
\hline DIG & $0.49 \pm 0.06$ & $0.10 \pm 0.03^{* *}$ & $0.51 \pm 0.07$ & $0.37 \pm 0.04$ \\
\hline \multicolumn{5}{|c|}{ Endocardial/epicardial ratio } \\
\hline CNT & $1.20 \pm 0.04$ & $0.46 \pm 0.10^{*}$ & $1.96 \pm 0.40^{*}$ & $0.99 \pm 0.12$ \\
\hline DIG & $1.19 \pm 0.07$ & $0.41 \pm 0.07^{* *}$ & $1.27 \pm 0.20$ & $0.84 \pm 0.14$ \\
\hline
\end{tabular}

Data are mean \pm SE; for all parameters, $n=9$.

$\mathrm{CNT}=$ Untreated control group; DIG = digoxin-pretreated group; LCx = left circumflex coronary artery; MBF $=$ myocardial blood flow .

${ }^{*} p<0.05$ and ${ }^{* *} p<0.01$ compared to appropriate baseline value within group (repeated-measures analysis of variance followed hy Dunnett's test for multiple comparisons). There were no significant difTerences between DIG and CNT groups during any time points.

Table III. Myocardial blood flow in nonischemic control area (left anterior distribution) at baseline, during left circumflex coronary artery occlusion, and after left circumflex coronary artery reperfusion

\begin{tabular}{|c|c|c|c|c|}
\hline & \multirow[b]{2}{*}{ Baseline } & \multirow{2}{*}{$\frac{\begin{array}{c}L C x \\
\text { occlusion }\end{array}}{50 \mathrm{~min}}$} & \multicolumn{2}{|c|}{$\begin{array}{c}L C x \\
\text { reperfusion }\end{array}$} \\
\hline & & & $1 \mathrm{hr}$ & $4 \mathrm{hr}$ \\
\hline \multicolumn{5}{|c|}{ Subendocardial MBF (ml/min/gm) } \\
\hline CNT & $0.70 \pm 0.06$ & $0.74 \pm 0.04$ & $0.59 \pm 0.04$ & $0.64 \pm 0.05$ \\
\hline DIG & $0.54 \pm 0.06$ & $0.56 \pm 0.04 \ddagger$ & $0.45 \pm 0.05 \dagger$ & $0.44 \pm 0.04 \dagger$ \\
\hline \multicolumn{5}{|c|}{ Midmyocardial MBF (ml/min/gm) } \\
\hline CNT & $0.67 \pm 0.08$ & $0.68+0.04$ & $0.52+0.03^{*}$ & $0.58 \pm 0.04$ \\
\hline DIG & $0.52 \pm 0.06$ & $0.56 \pm 0.04 \dagger$ & $0.48 \pm 0.04$ & $0.47 \pm 0.05$ \\
\hline \multicolumn{5}{|c|}{ Subepicardial MBF $(\mathrm{ml} / \mathrm{min} / \mathrm{gm})$} \\
\hline CNT & $0.52 \pm 0.06$ & $0.53 \pm 0.04$ & $0.41 \pm 0.03$ & $0.45 \pm 0.04$ \\
\hline DIG & $0.45 \pm 0.05$ & $0.48 \pm 0.04$ & $0.38 \pm 0.03$ & $0.37 \pm 0.03$ \\
\hline \multicolumn{5}{|c|}{ Endocardial/epicardial ratio } \\
\hline CNT & $1.42 \pm 0.05$ & $1.43 \pm 0.04$ & $1.47 \pm 0.05$ & $1.50 \pm 0.05$ \\
\hline DIG & $1.28 \perp 0.08$ & $1.28 \pm 0.08$ & $1.24 \perp 0.13$ & $1.28 \pm 0.09 t$ \\
\hline
\end{tabular}

Data are mean $\pm \mathrm{SE}$; for all parameters, $n=9$.

CNT = Untreated control group; DIG = digoxin-pretreated group; LCx = left circumflex coronary artery; MBF - myocardial blood tlow.

${ }^{*} p<0.05$ compared to appropriate baseline value within group (repeated-measures analysis of variance followed by Dunnett's test for multiple comparisons). $† p<0.05$ and $\ddagger p<0.01$ comparing DIG vs CNT parameter at specified time point (unpaired Student's $t$ test).

arterial pressure, rate-pressure product, and left circumflex coronary artery blood flow in each treatment group. The onset and degree of reduction in the aforementioned hemodynamic parameters tended to be earlier and greater, respectively, compared to appropriate baseline values within the digoxin-pretreated group compared to the control group (Table I).
Myocardial blood flow. Regional myocardial blood flow data from the ischemic left circumflex and nonischemic anterior areas are compared for the two treatment groups in Tables II and III, respectively. In the ischemic left circumflex region (Table II), there were no significant differences in regional blood flow between the digoxin-pretreated and control groups at baseline testing, during the period of 


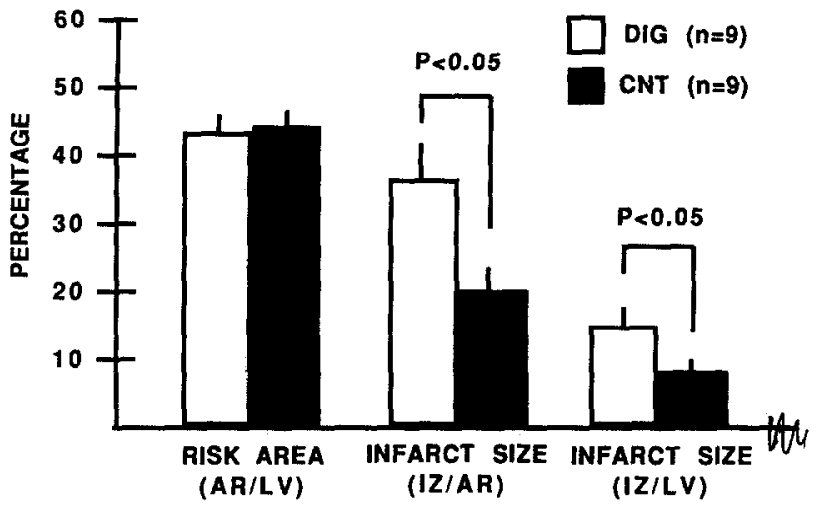

Fig. 1. Posterolateral myocardial infarct sizes in digoxinpretreated (DIG) and untreated control (CNT) animals after 60-minute occlusion of left circumflex coronary artery, followed by 6 hours of reperfusion. For both treatment groups, area at risk of infarction is expressed as percentage of left ventricle $(A R / L V)$; infarct zone size is expressed as percentages of area at risk (IZ/AR) and of left ventricle $(I Z / L V)$. Values are mean $\pm \mathrm{SE}$.

left circumflex coronary artery occlusion, and during coronary artery reperfusion, although there was a tendency for the blood flow in the digoxinpretreated group to be less than the control blood flow in the subepicardial tissue samples at baseline and during reperfusion. Similarly, there were no significant differences in endocardial/epicardial regional blood flow ratios between the digoxinpretreated and control groups in the left circumflex region at any time point tested, although the endocardial/epicardial ratio for the control group at 1 hour of left circumflex coronary artery reperfusion was markedly greater than the corresponding value in the digoxin-pretreated group $(+54 \%$ of digoxin pretreatment value, $p=0.14$ ) at this time point (Table II). Equivalent and significant reductions in regional myocardial blood flow occurred in all of the sampling regions within the digoxin-pretreated and control groups during the period of left circumflex coronary artery occlusion, suggesting the development of equal degrees of myocardial ischemia in the two treatment groups. At 4 hours after the commencement of left circumflex coronary artery reperfusion, subendocardial and midmyocardial blood flow was significantly reduced $(p<0.01$ for each sample area) relative to baseline values within the digoxin-pretreated group, whereas in the control group only subendocardial flow was reduced relative to baseline, and to a lesser extent than in the digoxin-pretreated group $(p<0.05)$. Endocardial/ epicardial ratios in the left circumflex regions for both the digoxin-pretreated and control groups were reduced significantly and equivalently during left

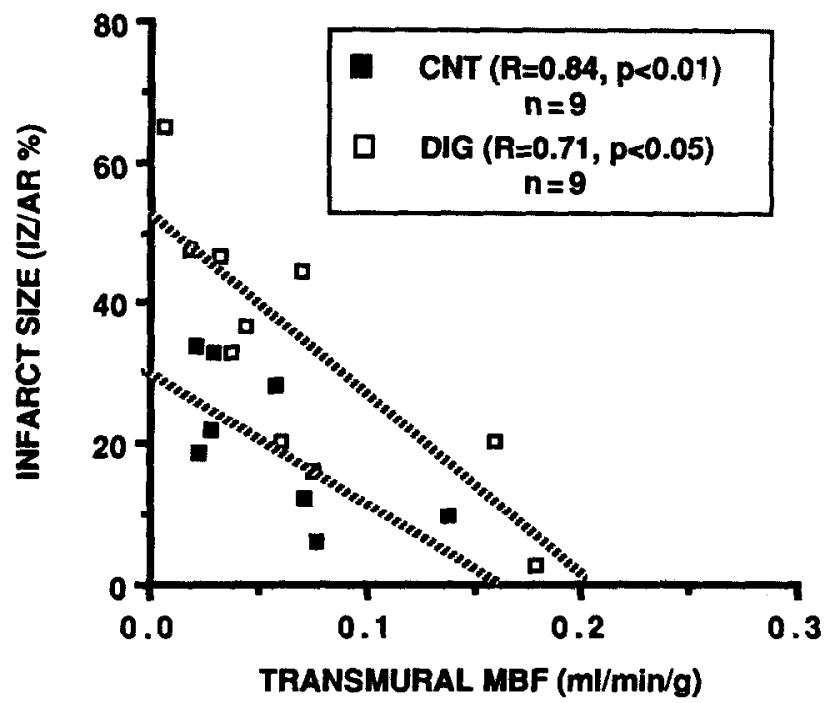

Fig. 2. Posterolateral myocardial infarct sizes (expressed as percentages of area at risk) for digoxin-pretreated (DIG, open squares) and control (CNT, closed squares) preparations plotted as function of transmural regional myocardial blood flow (MBF) in ischemic left circumflex coronary artery distribution at $\mathbf{5 0}$ minutes of coronary artery occlusion. Simple regression lines (dashed lines), correlation coefficients, and probability values are superimposed on graphs.

circumflex coronary artery occlusion, reflecting the relatively greater vulnerability of the subendocardium to blood flow reduction after coronary artery ligation in both groups. Within the control group, the endocardial/epicardial ratio in the left circumflex region was increased significantly relative to the baseline value at 1 hour after left circumflex coronary artery reperfusion, suggesting a marked enhancement in subendocardial blood flow early after reperfusion. A similar enhancement of subendocardial blood flow early after reperfusion was not observed in the digoxin-pretreated group (Table II).

In the nonischemic anterior region (Table III), there were no significant differences in regional myocardial blood flow between the two treatment groups at baseline testing, although blood flow in all three sampling regions tended to be reduced in the digoxin-pretreated group ( -14 to $-23 \%$ of control values) compared to the control group. Regional myocardial blood flow remained depressed in the digoxin-pretreated group relative to the control group during both the left circumflex coronary artery occlusion and reperfusion periods, with the values for regional blood flow in the digoxinpretreated group becoming significantly reduced in the subendocardial $(p<0.01)$ and midmyocardial 


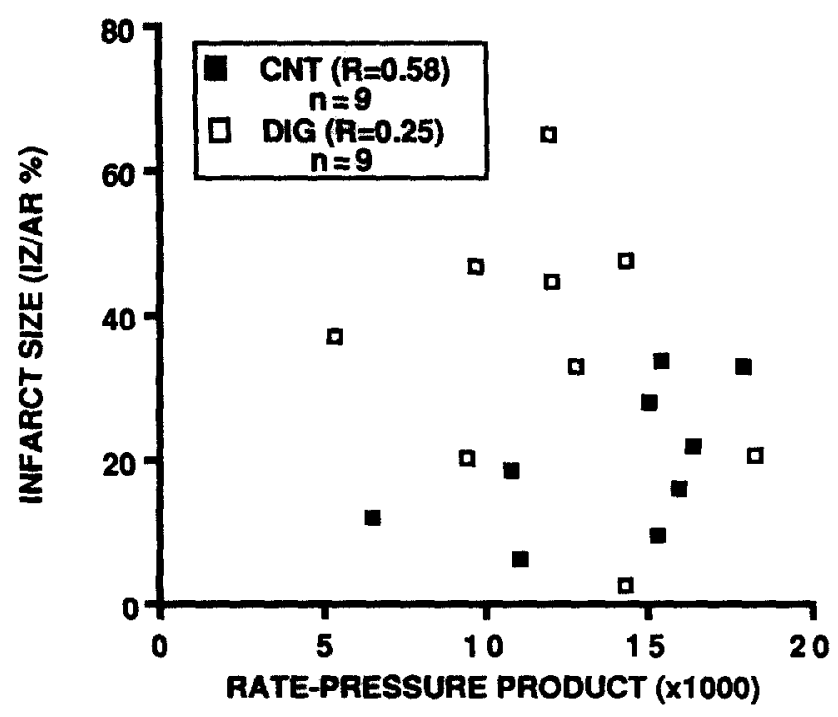

Fig. 3. Posterolateral myocardial infarct sizes (expressed as percentages of area at risk) for digoxin-pretreated (DIG, open squares) and control (CNT, closed squares) preparations plotted as function of rate-pressure product. Correlation coefficients for regression lines for both treatment groups (not shown) were not significant.

$(p<0.05)$ regions during left circumflex coronary artery occlusion, and in the subendocardial region at both 1 hour $(p<0.05)$ and 4 hours $(p<0.05)$ of left circumflex coronary artery reperfusion, compared to corresponding control values. In the nonischemic region, the only significant deviation from the baseline values for regional blood flow within either treatment group was a reduction in midmyocardial flow $(p<0.05)$ at 1 hour after left circumflex coronary artery reperfusion in the control group. Endocardial/epicardial ratios for the digoxin-pretreated group in the nonischemic area were consistently less at all time points relative to control values, with the digoxin-pretreated endocardial/epicardial blood flow ratio significantly reduced $(p<0.05)$ in comparison to the control value at 4 hours after left circumflex coronary artery reperfusion.

Size of myocardial infarction. The sizes of the developing myocardial infarctions for the digoxinpretreated and untreated control groups are shown in Fig. 1. For both groups, the infarct zones are quantitated as percentages of both the anatomic area at risk (IZ/AR) and the left ventricle (IZ/LV). The sizes of the risk areas for both groups are quantitated as percentages of the left ventricle (AR/LV). Posterolateral myocardial infarct size, expressed as a percentage of the area at risk, was $20.2 \pm 3.3 \%$ in untreated control animals vs $35.4 \pm$ $6.2 \%$ in the digoxin-pretreated group $(p<0.05)$. When the extent of ischemic injury was expressed as

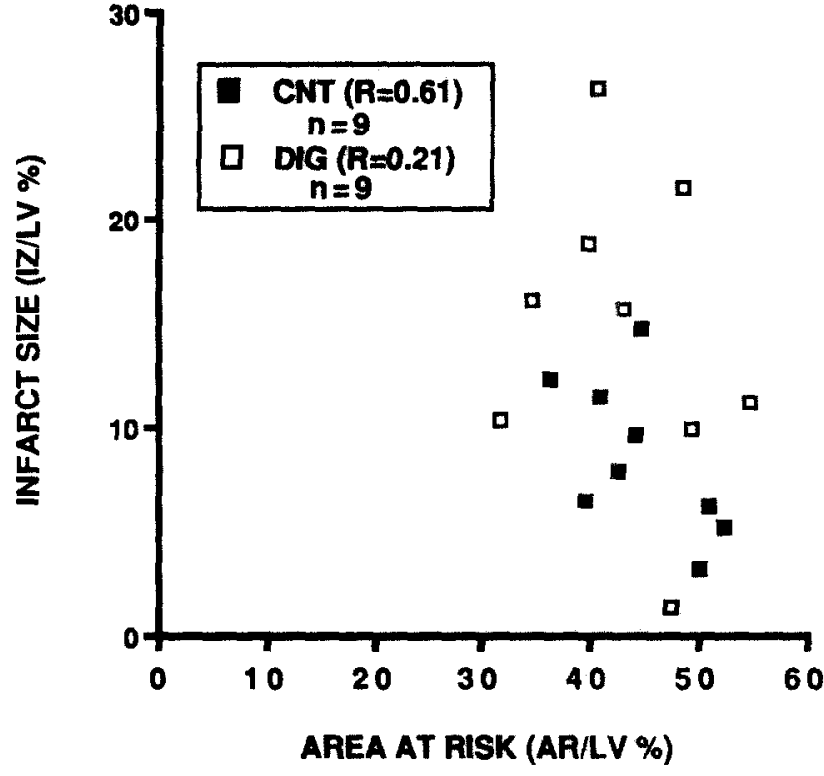

Fig. 4. Posterolateral myocardial infarct sizes (expressed as percentages of left ventricle) for digoxin-pretreated (DIG, open squares) and control (CNT, closed squares) preparations plotted as function of area at risk of infarction expressed as percentage of left ventricle). Correlation coefficients for regression lines for both treatment groups (not shown) were not significant.

a percentage of the left ventricle, infarct size was $8.6 \pm 1.3 \%$ in the control group vs $14.7 \pm 2.5 \%$ in the digoxin-pretreated group $(p<0.05)$. Regardless of the manner in which infarct size was quantitated, significantly larger myocardial infarctions developed within the left circumflex coronary distributions of digoxin-pretreated animals subjected to regional myocardial ischemia and reperfusion than in untreated control animals (Fig. 1). The anatomic areas at risk of infarction did not differ significantly between the digoxin-pretreated and untreated control animals subjected to regional posterolateral myocardial ischemia and reperfusion (Fig. 1).

Previous studies in this laboratory have used a maximum subepicardial ischemic collateral blood flow criterion of $0.2 \mathrm{ml} / \mathrm{min} / \mathrm{gm}$ during the period of left circumflex coronary artery occlusion to identify animals with "adequate" degrees of ischemia. The application of this collateral blood flow criterion to the present study would result in the exclusion of two digoxin-pretreated preparations with subepicardial collateral blood flows of 0.223 and $0.248 \mathrm{ml} /$ $\mathrm{min} / \mathrm{gm}$ during coronary artery occlusion, whereas no control untreated preparations would be excluded. Posterolateral myocardial infarct sizes in the animals not excluded by the collateral blood flow criterion are: (percentage of the area at risk) $20.2 \pm$ 
$3.3 \%$ in the control group vs $42.2 \pm 5.3 \%$ in the digoxin-pretreated group $(p<0.01)$ and (percentage of the left ventricle) $8.6 \pm 1.3 \%$ in the control group vs $17.1 \pm 2.2 \%$ in the digoxin-pretreated group $(p<0.01)$. Hence, significantly larger myocardial infarctions developed in the left circumflex coronary artery distributions of digoxin-pretreated animals subjected to regional myocardial ischemia and reperfusion than in untreated control animals regardless of the application of a maximal ischemic collateral blood flow criterion.

Determinants of myocardial infarct size. Linear regression analyses were performed to evaluate the influence of the size of the area at risk of infarction, rate-pressure product, and ischemic collateral blood flow on myocardial infarct size. Myocardial infarct sizes within both the digoxin-pretreated and control groups were correlated significantly with transmural regional myocardial blood flow in the central ischemic left circumflex distribution during coronary artery occlusion, with the infarct sizes in the digoxin-pretreated group being larger than those in the control group throughout the range of collateral blood flow values (Fig. 2). The infarct size-central ischemic regional blood flow regression lines for the two treatment groups were parallel, and the $95 \%$ confidence intervals did not overlap. In contrast, the myocardial infarct sizes in both treatment groups failed to correlate significantly with the rate-pressure product, an index of myocardial oxygen consumption (Fig. 3). Likewise, myocardial infarct sizes in the digoxin-pretreated and control groups failed to correlate significantly with the areas at risk of infarction, with the risk regions being relatively similar within and between groups (Fig. 4).

Development of ventricular arrhythmias. The frequency and complexity of ventricular ectopic complexes varied markedly within both treatment groups during coronary artery occlusion and reperfusion in this preparation; therefore, no attempt has been made to quantify and compare premature ventricular complex counts or grades of complexity. The incidence of ventricular fibrillation during the period of left circumflex coronary artery occlusion was two of nine $(22 \%)$ in the digoxin-pretreated group and none of nine $(0 \%)$ in the control group ( $p=$ not significant). Both of the digoxin-pretreated animals that developed ventricular fibrillation during left circumflex coronary artery occlusion underwent cardioversion by direct-current countershock. None of the animals in either treatment group had ventricular fibrillation during the reperfusion phase of this study.

\section{DISCUSSION}

Uncertainties regarding the potential detrimental actions of the cardiac glycosides in the setting of myocardial ischemic injury, particularly their influence on postinfarction mortality, ${ }^{16-21}$ persist despite the long-standing use of this class of drugs for inotropic support of the failing heart. The controversies surrounding the therapeutic use of cardiac glycosides during myocardial ischemia and the evolution of myocardial infarction reside primarily in the potential for exacerbation or facilitation of the development of ischemic ventricular arrhythmias, as well as a possible aggravation of myocardial ischemic injury resulting from unfavorable effects on myocardial oxygen demand, coronary blood flow, or both. ${ }^{22-24}$ Results of previous studies in this laboratory, with the use of canine models of myocardial ischemic injury, have shown a significant propensity for clinically relevant serum concentrations of digoxin to increase the incidence of ischemic postinfarction mortality ${ }^{1}$ and to promote the development of ischemic ventricular ectopic activity that is less sensitive to suppression with conventional antiarrhythmic agents. ${ }^{4}$ In the present study, pretreatment to achieve serum digoxin concentrations approximating the high range of therapeutic values resulted in an increase in the anatomic myocardial infarct size developing in response to a 60-minute period of regional myocardial ischemia followed by 6 hours of reperfusion in an anesthetized, open-chest canine preparation. An evaluation of the effects of digoxin on the hemodynamic and regional myocardial blood flow determinants of myocardial infarct size, as well as a comparison of the present results with those of previous studies, is presented below.

Hemodynamic effects of digoxin. The potential for digitalis to increase myocardial oxygen demand by virtue of a positive inotropic effect in the presence of a limited oxygen supply in the ischemic heart is central to the hypothesis that cardiac glycosides may exacerbate developing myocardial ischemic injury. ${ }^{22-24}$ However, the results of experimental assessments of the hemodynamic effects of acute digitalis administration during myocardial ischemia and acute myocardial infarction have yielded inconsistent results. Early experimental studies in anesthetized and conscious dogs showed no marked global hemodynamic effects with acutely administered cardiac glycosides during acute myocardial ischemia and subacutely after myocardial infarction, whereas global inotropic effects were seen 7 days after myocardial infarction. ${ }^{25,}{ }^{26}$ In anesthetized dogs, Banka et al. ${ }^{27}$ observed improvement in regional 
contractile function in nonischemic and "border zone" myocardial areas, but not in ischemic areas, with an acute intravenous bolus and maintenance infusion of digoxin administered at times varying from 15 minutes to 2 hours after coronary artery occlusion. In contrast, Vatner at el. ${ }^{28}$ reported significant increases in regional contractile function in nonischemic, moderately ischemic, and ischemic myocardial zones in conscious dogs receiving ouabain as an intravenous bolus injection 20 minutes after coronary artery occlusion. Recently, Banka et al. ${ }^{29}$ reported improvement in regional subendocardial and subepicardial contractile function in ischemic myocardium in anesthetized dogs receiving intravenous ouabain 30 minutes after partial coronary artery ligation ( $50 \%$ of baseline flow), whereas no improvement in regional function in ischemic tissue was noted with ouabain at 30 minutes after complete coronary artery ligation. It is noteworthy that no determinations of the metabolic costs associated with enhanced contractile performance produced by acute digitalis in moderately ischemic or ischemic tissues were performed in the previous evaluations of Vatner et al..$^{28}$ or Banka et al., ${ }^{29}$ and there was no assessment of the effects of digitalis on regional function or myocardial oxygen consumption during more prolonged periods of regional myocardial ischemia.

In the present study, the pretreatment of animals with digoxin for 6 to 7 days to achieve clinically relevant serum concentrations produced no marked effects on measured hemodynamic parameters at baseline testing after anesthesia and instrumentation compared to untreated control animals. Further, no striking differences between groups were noted during the periods of regional myocardial ischemia and reperfusion for parameters such as heart rate, left atrial and systemic arterial pressures, and rate-pressure product, with the exception of a slight decrease in mean arterial pressure in digoxinpretreated animals immediately after reperfusion of the ischemic myocardium. These hemodynamic data suggest no untoward effects of digoxin on the preload and afterload determinants of myocardial oxygen consumption that would account for an exacerbation of myocardial ischemic-reperfusion injury in this preparation, and these data are consistent with the failure of previous experimental evaluations (cited above) to demonstrate marked global hemodynamic effects with digitalis in canine models of myocardial ischemic injury. In the present study, the within-group reductions in mean systemic arterial pressure, rate-pressure product, and left circum- flex coronary artery blood flow observed during the reperfusion period in both treatment groups likely are a reflection of the development of myocardial necrosis in the area of myocardium rendered ischemic and reperfused.

Effects of digoxin on myocardial blood flow. Considerable experimental and clinical evidence indicates that cardiac glycosides possess direct and/or neurogenically mediated coronary vasoconstrictor activity $^{30}$ that may tend, in theory, to aggravate ischemic injury by limiting myocardial oxygen supply. $23,24,30$ In both conscious and anesthetized dogs, in the absence of myocardial ischemic injury, the acute administration of digitalis preparations results in increases in coronary ${ }^{31.33}$ and systemic ${ }^{34}$ vascular resistance. In a canine in situ isolated blood perfused heart preparation, Gross et al. ${ }^{31}$ observed increases in coronary vascular resistance and a redistribution of myocardial blood flow from the subendocardium to the subepicardium (i.e., an increased epicardial/endocardial blood flow ratio), which appeared to be dependent on concomitant effects on global contractile function after the acute intravenous administration of ouabain. The effects of digitalis on regional myocardial blood flow during myocardial ischemia and infarction, however, are less well characterized. Sagar et $a .^{35}$ reported increases in coronary vascular resistance, in some instances resulting in ventricular fibrillation, after the acute intravenous administration of acetylstrophanthidin or digoxin during acute global myocardial ischemia in anesthetized dogs, whereas Vatner et al. ${ }^{28}$ observed increases in regional blood flow to normal, moderately ischemic, and ischemic regions after the acute intravenous administration of ouabain after 20 minutes of coronary artery occlusion in conscious dogs.

In the present study, absolute values for regional myocardial blood flow did not differ significantly between the digoxin-pretreated and control animals at baseline, although regional blood flow in both the anterior nonischemic and posterolateral ischemic areas, particularly in the subendocardial sections, tended to be decreased in the digoxin-pretreated group relative to the control group. In the left circumflex coronary artery distribution, subendocardial and midmyocardial regional blood flow was reduced to a greater extent within the digoxinpretreated group compared to that within the control group during late reperfusion. In the nonischemic left anterior distribution, absolute regional myocardial blood flow values were significantly lower in the digoxin-pretreated group during left cir- 
cumflex coronary artery occlusion (subendocardial and midmyocardial) and reperfusion (subendocardium at 1 and 4 hours) compared to the control group. Left circumflex coronary artery blood flow was also reduced earlier and to a greater degree during the late reperfusion period in the digoxinpretreated group compared to the control group. Finally, the endocardial/epicardial blood flow ratios tended to be lower in the digoxin-preteated group than in the control group, both in the left circumflex distribution (during early and late reperfusion) and in the nonischemic anterior region (all time points), suggesting less coronary blood flow supply to the subendocardial region in digoxin-pretreated animals, which is consonant with the findings of Gross et al. $^{31}$ The preceding findings suggest that pretreatment with digoxin may have limited regional coronary blood flow to the subendocardium, which inherently is more vulnerable to myocardial ischemic injury than other areas of the myocardium ${ }^{36}$; it may also have reduced the amount of available collateral coronary blood flow from the nonischemic region to the border areas of the ischemic zone. Either of the aforementioned effects may have contributed to an extension of myocardial infarct size in the present study. It must be noted, however, that the potential influence of the evolving posterolateral myocardial infarctions must be considered in the interpretation of the regional blood flow data. Alterations in regional myocardial blood flow in the digoxin-pretreated animals, particularly in the left circumflex distribution during the late reperfusion period, may be caused by the development of significantly larger myocardial infarctions in this region compared to control preparations.

Effect of digoxin on myocardial ischemic injury. Relatively few experimental studies have directly evaluated the effect of digitalis on the severity or extent of an evolving myocardial infarction. In anesthetized dogs, Maroko et al..$^{37}$ observed an increase in the extent and severity of acute myocardial ischemia, as reflected in the distribution and amplitude of epicardial electrograms, in response to the acute intravenous administration of ouabain immediately preceding a 20-minute coronary artery ligation. With the use of the same preparation, however, Watanabe et al.$^{38}$ reported that the acute intravenous administration of ouabain to anesthetized dogs with acute pentobarbital-induced left ventricular failure caused a reduction in the extent and severity of temporary acute myocardial ischemia. In anesthetized dogs subjected to serial 10-minute coronary artery occlusions, Rude et al. ${ }^{39}$ concluded that moderate inotropic stimulation with intravenous bolus injections of ouabain did not increase the severity of the brief episodes of regional ischemia, provided that neither heart rate nor rate-pressure product was increased. In a recent report, Alker and Kloner ${ }^{40}$ demonstrated that an intravenous bolus administration of digoxin early during a complete 6-hour coronary artery ligation in anesthetized dogs failed to alter the size of the resulting myocardial infarct compared to vehicle-treated control dogs. However, the myocardial infarct sizes attained in the vehicleand digoxin-treated animals in the complete coronary ligation model approximated $90 \%$ of the area at risk of infarction, suggesting little possibility to demonstrate a significant extension of necrotic damage with an intervention. ${ }^{40}$

In the present investigation, the effects of digoxin pretreatment on the extent of evolving myocardial infarction were evaluated in an anesthetized canine preparation subjected to 60 minutes of regional myocardial ischemia, followed by reperfusion of the ischemic area for 6 hours. The duration of myocardial ischemic injury used in the present study has been shown by Eng and et al..$^{36}$ to correspond to a rapid period of progression of myocardial necrosis in the dog. Therefore, the 60-minute coronary artery occlusion period should be optimum for demonstrating the ability of an intervention to increase/accelerate or decrease/retard the extent of evolving myocardial ischemic injury. In this study, pretreatment with digoxin to yield serum concentrations in the upper range of clinically therapeutic values was associated with significant increases in anatomic infarct size after regional myocardial ischemicreperfusion injury compared to untreated control preparations. Myocardial infarct sizes in both the digoxin-pretreated and untreated control animals were correlated most significantly with regional myocardial blood flow during ischemia. Previous investigators have stressed the dependence of evolving myocardial infarct size on the magnitude of ischemic collateral blood flow. ${ }^{7}{ }^{14}$ For both treatment groups in the present study, the regression lines for infarct size and ischemic blood flow were parallel and did not overlap, indicating that a significant difference in evolving myocardial infarct sizes existed between the two groups, but this difference was not attributable to differences in the magnitude of collateral flow in the central ischemic area (i.e., equivalent degrees of ischemia had been attained in the two groups). The anatomic area at risk of infarction was not altered by digoxin pretreatment in the present study; neither did myocardial infarct sizes in either treatment group correlate significantly with area at risk. The reported degree of relation- 
ship between infarct size and area at risk has varied in previous investigations, 7,14 with the failure of infarct size to correlate significantly with area at risk in one previous study having been attributed to a relatively narrow range of risk regions evaluated. ${ }^{8} \mathrm{~A}$ similarly narrow range of areas at risk of infarction was encountered in the present study.

Conclusions. Pretreatment with digoxin to achieve serum concentrations in the upper range of clinically therapeutic values was associated with a significant increase in posterolateral myocardial infarct size in anesthetized dogs subjected to 60 minutes of left circumflex coronary artery occlusion, followed by 6 hours of reperfusion. Regional myocardial blood flow tended to be reduced in digoxin-pretreated dogs compared to untreated control animals during baseline testing and was significantly reduced in anterior subendocardial sites in digoxin-pretreated animals during regional myocardial ischemia and reperfusion, suggesting that digoxin may have limited subendocardial coronary blood flow. No marked global hemodynamic differences were noted between digoxin-pretreated and untreated control animals that could account for a significantly enhanced myocardial oxygen demand in the presence of digoxin. Further studies with the use of estimates of regional contractile function and myocardial oxygen supply and demand are required to characterize the metabolic cost of therapeutic digoxin during regional myocardial ischemic injury. These findings suggest that an exacerbation of evolving myocardial ischemic injury, resulting in an increase in infarct size, may occur in the presence of clinically relevant serum digoxin concentrations.

\section{REFERENCES}

1. Lynch JJ, Montgomery DG, Lucchesi BR. Facilitation of lethal ventricular arrhythmias by therapeutic digoxin in conscious postinfarction dogs. AM HEART J 1986;111:883.

2. Nelson SD, MacEwen S, Lucchesi BR, Lynch JJ. Antiarrhythmic actions of left stellectomy in digitalis-mediated malignant ventricular arrhythmias in the post infarcted canine heart. J Cardiovasc Pharmacol. (In press.)

3. Lynch JJ, Kitzen JM, Hoff PT, Lucchesi BR. Reduction in digitalis-associated postinfarction mortality with nadolol in conscious dogs. AM HEART J. (In press.)

4. Lynch JJ, Lucchesi BR. Effect of digoxin on the extent of injury and the severity of arrhythmias during acute myocardial ischemia and infarction in the dog. J Cardiovasc Pharmacol 1988;11:193.

5. Sheehan F, Epstein S. Determinants of arrhythmic death due to coronary spasm: effect of preexisting coronary artery stenosis on the incidence of reperfusion arrhythmias. Circulation 1981;65:259.

6. Jolly SR, Kane WJ, Bailie MB, Lucchesi BR. Canine myocardial reperfusion injury: its reduction by the combined administration of superoxide dismutase and catalase. Circ Res $1984 ; 54: 277$

7. Reimer KA, Jennings RB, Cobb FR, et al. Animal models for protecting ischemic myocardium: results of the NIHLBI cooperative study. Comparison of unconscious and conscious dog models. Circ Res 1985;56:651.

8. Gallagher KP, Buda AJ, Pace D, Gerren RA, Shlafer M. Failure of superoxide dismutase and catalase to alter size of infarction in conscious dogs after 3 hours of occlusion followed by reperfusion. Circulation 1986:73:1065.

9. Fishbein MC, Meerbaum S, Rit J, et al. Early phase acute myocardial infarct size determination: validation of the triphenyltetrazolium chloride tissue enzyme staining technique. AM HEART J 1981;101:593.

10. Barnard RJ, Okamoto F, Buckberg GD, et al. Studies of controlled reperfusion after ischemia. III. Histochemical studies: inability of triphenyltetrazolium chloride nonstaining to define tissue necrosis. J Thorac Cardiovasc Surg $1986 ; 92: 502$

11. Romson JL, Bush LR, Jolly SR, Lucchesi BR. Cardioprotective effects of ibuprofen in experimental regional and global myocardial ischemia. $J$ Cardiovasc Pharmacol 1982;4:187.

12. Kirlin PC, Romson JL, Pitt B, Abrams GD, Schork MA, Lucchesi BR. Ibuprofen-mediated infarct size reduction. Effects on regional myocardial function in canine myocardial infarction. Am J Cardiol 1982;50:849.

13. Heymann MA, Payne BD, Hoffman JIE, Rudolph AM. Blood flow measurements with radionuclide-labeled particles. Prog Cardiovasc Dis 1977;20:55.

14. Muira T, Yellon DM, Hearse DJ, Downey .JM. Determinants of infarct size during permanent occlusion of a coronary artery in the closed chest dog. I Am Coll Cardiol 1987; $9: 647$.

15. Gobel FL, Nordstrom LA, Nelson RR, Jorgensen CR, Wang $\mathrm{Y}$. The rate-pressure product as an index of myocardial oxygen consumption during exercise in patients with angina pectoris. Circulation 1978;57:549.

16. Moss AJ, Davis HT, Conard DL, DeCamilla dJ, Odoroff CL. Digitalis-associated cardiac mortality after myocardial infarction. Circulation 1980;64:1150.

17. Madsen EB, Gilpin E, Henning H, et al. Prognostic impor tance of digitalis after acute myocardial infarction. $J$ Am Coll Cardiol 1984;3:681.

18. Bigger JT, Fleiss FL, Rolnitzky LM, Merab JP, Ferrick KP. Effect of digitalis treatment on survival after acute myocardial infarction. Am $J$ Cardiol 1985;55:623.

19. Byington R, Goldstein S, for the BHAT Research Group. Association of digitalis therapy with mortality in survivors of acute myocardial infarction: observations in the Beta-Blocker Heart Attack Trial. J Am Coll Cardiol 1985;6:976.

20. Goldstein S, Landis JR, Leighton R, et al. Predictive survival models for resuscitated victims of out-of-hospital cardiac arrest with coronary heart disease. Circulation 1985;71:873.

21. Muller JE, Turi ZG, Stone PH, et al, and the MILIS Study Group. Digoxin therapy and mortality after myocardial infarction. Experience in the MILIS study. N Engl $J$ Med $1986 ; 314: 265$

22. Rahimtoola SH, Gunnar RM. Digitalis in acute myocardial infarction: Help or hazard? Ann Intern Med 1975;82:234.

23. Marcus FI. Use of digitalis in acute myocardial infarction Circulation 1980;62:17

24. Karliner JS, Braunwald E. Present status of digitalis treatment of acute myocardial infarction. Circulation 1972; 45:891.

25. Hood WB, McCarthy B, Lown B. Myocardial infarction following coronary ligation in dogs. Hemodynamic effects of isoproterenol and acetylstrophanthidin. Circ Res 1967; 21:191.

26. Kumar R, Hood WB, Joison J, Gilmour DP, Norman JC, Abelmann WH. Experimental myocardial infarction. VI. Efficacy and toxicity of digitalis in acute and healing phase in intact conscious dogs. J Clin Invest 1970;49:358.

27. Banka VS, Chadda KD, Bodenheimer MM, Helfant RH. Digitalis in experimental acute myocardial infarction. Differ ential effects on contractile performance of ischemic, border, 
and nonischemic ventricular zones in the dog. Am J Cardiol 1975;35:801.

28. Vatner SF, Baig H, Manders WT, Murray PA. Effects of a cardiac glycoside on regional function, blood flow, and electrograms in conscious dogs with myocardial ischemia. Circ Res 1978;43:413.

29. Banka VS, Yamazaki H, Agarwal JB, Bodenheimer MM, Helfant RH. Effects of digitalis on subendocardial and subepicardial dysfunction during acute ischemia. Circulation 1982;65:1315.

30. Tanz RD. Possible contribution of digitalis-induced coronary constriction to toxicity. AM HEART J 1986;111:812.

31. Gross GJ, Waltier DC, Hardman HF, Somani P. The effect of ouabain on nutritional circulation and regional myocardial blood flow. Am HearT J 1977;93:487.

32. Vatner SF, Higgins CB, Franklin D, Braunwald E. Effects of a digitalis glycoside on coronary and systemic hemodynamics in conscious dogs. Circ Res 1971;28:470.

33. Hamlin NP, Willerson JT, Garan H, Powell WJ. The neurogenic vasoconstrictor effect of digitalis on coronary vascular resistance. J Clin Invest 1974;53:288.

34. Higgins CB, Vatner SF, Braunwald E. Regional hemodynam. ic effects of a digitalis glycoside in the conscious dog with and without experimental heart failure. Circ Res 1972;30:406.
35. Sagar KB, Hanson EC, Powell WJ. Neurogenic coronary vasoconstrictor effects of digitalis during acute global ischemia in dogs. $J$ Clin Invest 1977;60:1248.

36. Eng C, Sangho C, Factor SM, Kirk ES. A nonflow basis for the vulnerability of the subendocardium. J Am Coll Cardiol 1987;9:374.

37. Maroko PR, Kjekshus JK, Sobel BE, et al. Factors influencing infarct size following experimental coronary artery occlusion. Circulation 1971;43:67.

38. Watanabe T, Covell JW, Maroko PR, Braunwald E, Ross JR. Effects of increased arterial pressure and positive inotropic agents on the severity of myocardial ischemia in the acutely depressed heart. Am J Cardiol 1972;30:371.

39. Rude RE, Izquierdo C, Bush LR, Buja LM, Willerson JT. Effects of inotropic and chronotropic stimuli on acute myo. cardial ischemic injury. II. Studies with dopamine and ouabain in the barbiturate-anesthetized dog. J Cardiovasc Pharmacol 1983;5:717.

40. Alker KJ, Kloner RA. The effect of digitalis on experimental myocardial infarct size and hemodynamics. AM HEART J 1987;113:1353.

\title{
Management of patients with prolonged ischemic chest pain without acute myocardial
}

\section{infarction}

\begin{abstract}
Consecutive patients admitted to a Coronary Care Unit during 1981-1982 were studied. One hundred twenty-elght patients had prolonged ischemic chest pain without developing myocardial infarction. Follow-up information was available in 121 at an average period of 38 months. Thirty-eight patients $(32.8 \%)$ sustained significant cardiovascular events, including 14 cardiac deaths. Only 15 patients were symptom-free off treatment. Prognosis was best determined from the resting ECG. Transient ST-T wave shifts predicted patients at high risk of further cardlovascular events and allowed selection of a group meriting more intensive treatment. (AM HEART J 1988; 115:1182.)
\end{abstract}

\section{J. A. Wilson, M.R.C.P., and J. B. Irving, B.Sc., F.R.C.P. West Lothian, Scotland}

Unstable angina is an imprecise term describing a symptom complex intensively investigated by cardiologists in the last 10 to 15 years. The dilemmas in the management of patients with these symptoms

From the Medical Unit, Bangour General Hospital.

Received for publication Sept. 3, 1987; accepted Feb. 1, 1988.

Reprint requests: Dr. J. B. Irving, Medical Unit, Bangour General Hospital, Broxburn, West Lothian EH52 6LR, Scotland, United Kingdom. have been reviewed recently. ${ }^{1}$ Guidelines for treatment in large hospitals, with ready access to arteriography, angioplasty, and surgery, are not applicable to smaller hospitals, remote from such facilities. This study was designed to identify patients who merited referral for urgent investigation from a general hospital admitting approximately 650 patients per annum to a four-bed Coronary Care Unit (CCU). The study was limited to a subgroup of "unstable angina" patients admitted with prolonged 